\title{
Catechol as a New Electron Hot Spot of Carbon Nitride
}

\author{
Kimoon Lee ${ }^{\dagger}$, Jeonga Kim ${ }^{\dagger}$, Saehan Choi, Nyeongbeen Jo, and Yoon Sung Nam*
}

Department of Materials Science and Engineering, Korea Advanced Institute of Science and Technology, 291 Daehak-ro, Yuseong-gu, Daejeon, 34141, Republic of Korea

${ }^{\dagger}$ These authors equally contributed to this work

\begin{abstract}
Graphitic carbon nitride $(\mathrm{CNx})$ is a promising photocatalyst with visible-light sensitivity, attractive band-edge positions, tunable electronic structure, and eco-friendliness. However, their applications are limited by a low catalytic activity due to inefficient charge separation and insufficient visiblelight absorption. Here we show a new method to generate the electron polarization of $\mathrm{CNx}$ toward the edge via the chemical conjugation of catechol to $\mathrm{CNx}$ for enhanced photochemical activity. The electron-attracting property of catechol/quinone pairs induces the accumulation of photoexcited electrons at the edge of conjugated catechol-CNx hybrid nanostructure (Cat-CNx), , serving as an electron hot spot, as demonstrated by positive open-circuit photovoltage, which increases electron transfer through the conjugated catechol while suppressing charge recombination in the $\mathrm{CNx}$. The catechol conjugation also widens the photoactive spectrum via the larger range delocalization of $\pi$-electrons. Accordingly, Cat-CNx reveals a $6.3 \times$ higher reductive photocurrent density than CNx. Gold ion reduction dramatically increased due to the enhanced electron transfer activity of Cat-CNx in cooperation with the inherent hydrophilicity and metal chelating property of catechols. Cat-CNx exhibits a $4.3 \times$ higher maximum adsorption capacity for gold ions under simulated sun light illumination compared to $\mathrm{CNx}$. This work suggests that the
\end{abstract}


post-modification of CNx's $\pi$-conjugated system is a promising route to handle varied shortcomings and broaden availability of $\mathrm{CNx}$.

KEYWORDS: graphitic carbon nitride; catechol chemistry; photocatalyst; urban gold mining

\section{Introduction}

Carbon nitride, denoted as $\mathrm{CNx}$, has received considerable attention due to its metal-free semiconducting properties, visible-light photoactivity, earth abundance, and excellent thermal and chemical stability. ${ }^{1,2}$ Both the conduction and valence band-edge positions of $\mathrm{CNx}$ are desirable to produce a sufficient thermodynamic driving force for various redox reactions including hydrogen evolution, oxygen evolution, and carbon dioxide reduction. ${ }^{1,3-7}$. CNx is particularly attractive for photocatalytic reduction due to the higher conduction band-edge position of $\mathrm{CNx}$ in comparison to other semiconductors.

Despite the multiple attracting characteristics, the wide photocatalytic applications of $\mathrm{CNx}$ are limited by several drawbacks. First of all, $\mathrm{CNx}$ still suffers from the limited absorption of visible light due to its bandgap energy insufficient for full solar spectrum absorption and low separation efficiency of photo-induced charge carriers. ${ }^{8}$ Secondly, the innate n-type characteristics of $\mathrm{CNx}$ impedes efficient electron transfer to a neighboring redox couple due to an internal electric field generated at the interface despite the high conduction band edge position. ${ }^{9}$ Finally, the innate hydrophobic texture of $\mathrm{CNx}$ limits its use in aqueous media, which also causes poor water-based catalytic reactions with polar redox couples. ${ }^{10}$

Diverse strategies have been attempted to tune the photochemical properties, such as the recombination rate and photoactive spectrum, of $\mathrm{CNx}$ by introducing heterogeneous atoms and 
vacancy defects to modify the constituents of the heptazine and triazine repeating units. ${ }^{11-19}$ Particularly, conjugating organic molecules functionalized to $\mathrm{CNx}$ during synthesis have shown high potentials for increasing the photoactivity of $\mathrm{CNx}$ by extending the $\pi$-conjugated structure of $\mathrm{CNx}^{20-23}$ However, few studies have explored the post-synthetic functionalization of $\mathrm{CNx}$ to modify the photochemical properties of $\mathrm{CNx}$. The conjugation of organic molecules through postsynthetic modification is advantageous because it allows to utilize the chemical properties of functional groups of introduced organic molecules as well as tuned photoactivity by the modified chemical structure. ${ }^{24}$ For instance, Zhang et al. reported the modified photochemical properties of $\mathrm{CNx}$ via post-modification by conjugating various functional groups within the $\mathrm{C}-\mathrm{N}$ heterocyclic repeating units, though they did not fully scrutinize the detailed variations of photochemical properties of $\mathrm{CNx}$ after the post-modification such as band structures and photo-induced charges' behaviors using various characterizations. Moreover, they did not track how chemical properties of functional group could impact on photocatalytic applications. ${ }^{24}$ In addition, the conjugation of functional molecules within the $\mathrm{C}-\mathrm{N}$ heterocyclic repeating units can more facilitate the separation of photo-induced charges produced within the repeating units compared to post-modification to terminal amine groups. Previous studies about the post-modification of $\mathrm{CNx}$ utilizing the reactivity of the terminal amine groups showed small photoluminescence quenching implying limited charge separation with little increment of photoactivity in visible-light region. ${ }^{25,26}$

Among numerous possible organic molecules that can be conjugated to $\mathrm{CNx}$, a catechol moiety, found in bio-inspired adhesives, has an outstanding potential to solve the various drawbacks of $\mathrm{CNx}$ due to its versatility. ${ }^{27-31}$ When covalently introduced to the $\mathrm{CNx}$ 's $\pi$ conjugated system, the aryl component of catechols can extend the $\pi$-conjugation of $\mathrm{CNx}$, and the hydroxyl groups increases the hydrophilicity of $\mathrm{CNx}$ enabling applications in aqueous media. ${ }^{24,32}$ 
Notably, previous studies reported that catechol/quinone pairs introduced via polydopamine coatings on $\mathrm{CNx}$ provided electron-attracting properties leading to the facilitated extraction of photo-induced electrons from $\mathrm{CNx}$ and alleviation of the restricted electron transfer to redox couples. ${ }^{33,34}$ However, this approach has several drawbacks when $\mathrm{CNx}$ is used as a photocatalyst for a reduction reaction compared to conjugating catechol groups in a single molecular form. Firstly, given that the conformally coated layer of polydopamine might act as a diffusion barrier to hole scavengers that must diffuse to the reactive sites of $\mathrm{CNx}$ to consume photo-induced holes, the exposure of reactive sites for the hole consumption via the single-molecular attachment allows to avoid a bottle-neck effect caused by the accumulation of holes. ${ }^{19,35}$ In addition, unless carefully controlling a self-polymerization process for polydopamine coatings, a thick polydopamine layer, showing a wide absorption of visible light, can drastically decrease the light intensity irradiating to $\mathrm{CNx}$, resulting in the small number of photo-induced charges in $\mathrm{CNx}$ and subsequently limited photocatalytic power by $\mathrm{CNx} .{ }^{36}$

Our recent work demonstrated that the adsorption and photochemical reduction of gold ions by polyphenols is a promising strategy for eco-friendly urban gold mining from electronic wastes to satisfy the high demand for gold by achieving large adsorption capacity. ${ }^{29,37}$ The redox chemistry of catechol, where catechol is oxidized to semiquinone and quinone through the protoncoupled electron transfer, allows the chelation and reduction of gold ions. In this context, catecholconjugated $\mathrm{CNx}$ can be an attractive adsorbent for urban gold mining owing to the desirable conduction band-edge positions of $\mathrm{CNx}$ for large thermodynamic driving force for the reduction of metal ions in collaboration with the innate catechol chemistry. Furthermore, the metal-free and low-cost characteristics of catechol-conjugated $\mathrm{CNx}$ make it attractive as a cost-effective way to recover the value-added metal from electronic wastes. 
Here, we demonstrate the enhanced photocatalytic performance of conjugated catechol$\mathrm{CNx}$ hybrids for reduction reactions. Catechols were introduced in a single-molecular form within the heptazine and triazine repeating units of $\mathrm{CNx}$, driving the modification of photochemical properties of CNx. The conjugated catechol-CNx hybrids showed upshifts of both conduction and valence band edges and improved photo-induced charge carriers' separation as demonstrated by photoluminescence quenching. The conjugated catechol-CNx hybrids also exhibited the enhanced photocurrent density for a photoelectrochemical reduction reaction. The improved photocatalytic activity is attributed to the modified surface properties enabling the photo-induced electrons to accumulate at the surface rather than be swept to the bulk phase of CNx. Furthermore, the photoenhanced reductive activity of catechol-CNx hybrids was further utilized for urban gold mining to achieve high adsorption capacity by harnessing photo-induced electrons to reduce gold ions.

\section{Results and Discussion}

Catechol-functionalized Graphitic Carbon Nitride. The preparation procedures of CNx and catechol-functionalized graphitic carbon nitride denoted as Cat-CNx, are described in Scheme 1. Briefly, $\mathrm{CNx}$ was synthesized through the pyrolysis of dicyandiamide as a nitrogen-containing precursor. Catechol was conjugated to the synthesized $\mathrm{CNx}$ according to the Prato's reaction, the 1,3-dipolar cycloaddition of azomethine ylides with 3,4-dihydroxybenzaldehyde. ${ }^{24}$ The carbon-tonitrogen $(\mathrm{C} / \mathrm{N})$ molar ratio was determined using elemental analysis (EA) to confirm the introduction of a heterogenous functional group (Table 1). ${ }^{24}$ The $\mathrm{C} / \mathrm{N}$ ratio of the as-synthesized $\mathrm{CNx}$ was 0.68 , lower than the theoretical value of 0.75 , indicating that partially polymerized structures exist in the as-synthesized $\mathrm{CNx}^{38}$ After the Prato's reaction, Cat-CNx exhibits an 
increased $\mathrm{C} / \mathrm{N}$ ratio $(0.74)$, which indicates the introduction of carbon-rich moieties. The chemical structure of $\mathrm{CNx}$ was examined using ${ }^{13} \mathrm{C}$ cross-polarization magic angle spinning nuclear magnetic resonance $\left({ }^{13} \mathrm{C}\right.$ CP-MAS-NMR) (Figure 1A) to confirm whether the increased $\mathrm{C} / \mathrm{N}$ ratio is ascribed to the introduction of catechols. Two characteristic peaks of $\mathrm{CNx}$ at 167.2 and 159.0 ppm, corresponding to $\mathrm{CN}_{3}$ and $\mathrm{CN}_{2}\left(\mathrm{NH}_{\mathrm{x}}\right)$, respectively, were found in the NMR spectrum of Cat$\mathrm{CNx}^{9,39}$ The broad peak at $130.8 \mathrm{ppm}$ corresponds to the catechol group, showing a similar peak position when catechols bound in their single molecular form on metal oxides in previous studies. ${ }^{40,41}$ Notably, other peaks except 130.8 ppm that could be observed in the previous studies are not discernible because of the low signal to noise ratio in our characterization presumably arising from the low amount of the introduced catechols, evidenced by thermogravimetric analysis (TGA) (vide infra), or the lack of Brownian motion required to eliminate specific interaction. ${ }^{42}$ However, the peaks at $46.4 \mathrm{ppm}(-\mathrm{NCN}-), 28.9 \mathrm{ppm}\left(-\mathrm{NCH}_{2}\right)$, and $24.9 \mathrm{ppm}\left(-\mathrm{CH}_{3}\right)$, which are only detected in the NMR spectrum of Cat-CNx, support the presence of the covalent linkage of CNx with catechol by the Prato's reaction, as found in a previous report. ${ }^{43}$ Barrett et al. suggested that polyphenolic precursors had an ability to form coating layers by following a similar path with self-polymerization of dopamine through deprotonation and autoxidation to form reactive quinones. ${ }^{45}$ However, although previously reported ${ }^{13} \mathrm{C}$ CP-MAS-NMR spectra of polyaromatic components showed broad and ambiguous peaks between $150 \mathrm{ppm}$ and $100 \mathrm{ppm}$, the NMR spectrum of Cat-CNx showed the absence of the ambiguous peaks, inferring that the selfpolymerization of 3,4-dihydroxybenzaldehyde did not occur or occur to a negligible level under the present experimental conditions. ${ }^{42,46}$ 
Braunauer-Emmett-Teller (BET) surface area analysis exhibited the specific surface areas of $12.02 \mathrm{~m}^{2} \mathrm{~g}^{-1}$ and $10.76 \mathrm{~m}^{2} \mathrm{~g}^{-1}$ for $\mathrm{CNx}$ and Cat-CNx, respectively (Figure $\mathbf{S 1}$ and Table 1). The catechol conjugation only slightly reduced the specific surface area of $\mathrm{CNx}$, indicating that neither aggregates nor the structural distortion of $\mathrm{CNx}$ might occur during the chemical modification. ${ }^{37}$ TGA for $\mathrm{CNx}$ and Cat-CNx was conducted to quantify the number of conjugated catechols, showing an additional weight percentage change of $0.64 \%$ for Cat-CNx (Figure S2). One catechol group was attached per $\sim 100$ heptazine repeating units, calculated by the residual mass, and given the BET surface area and Avogadro's number, two catechol groups per unit surface area $\left(\mathrm{nm}^{2}\right)$ of Cat-CNx were conjugated (see Supporting Information). ${ }^{24,44}$ The low weight percentage by the introduction of catechols further supports the negligible self-polymerization of 3,4dihydroxybenzaldehyde.

The crystallinity of CNx and Cat-CNx was examined using X-ray diffraction (XRD) analysis (Figure 1B). Two characteristic peaks were detected in the XRD patterns of CNx and Cat$\mathrm{CNx}$ at $2 \theta=13.3^{\circ}$ and $27.3^{\circ}$, originating from inner planar crystallinity (the (100) plane), and interlayer stacking (the (002) plane), respectively. Both $\mathrm{CNx}$ and Cat-CNx have an interplanar distance of $3.35 \AA$, as determined by the peak position, consistent with a previously reported value.$^{47}$ No marked variation in the peak positions between $\mathrm{CNx}$ and Cat-CNx indicates that the crystallinity of $\mathrm{CNx}$ was maintained without significant distortion after the conjugation of catechols. The scanning electron microscopy (SEM), bright-field transmission electron microscopy (TEM), and scanning TEM (STEM) images of CNx and Cat-CNx exhibit rough and sheet-like layered morphologies, typically observed for $\mathrm{CNx}$, and micro-sized particles with irregular shapes (Figures 1C, 1D and S3-5).$^{48}$ No structural alteration was caused by the 
conjugation of catechol groups to CNx. Fourier Transform-Infrared (FT-IR) spectra of CNx and Cat-CNx exhibited peaks in the $1200-1650 \mathrm{~cm}^{-1}$ region and a peak at $809 \mathrm{~cm}^{-1}$, representing the stretching modes of $\mathrm{CN}$ heterocycles and the breathing modes of triazine units, respectively (Figure S6). ${ }^{49}$ The results corroborate that the Prato's reaction with 3,4-dihydroxybenzaldehyde caused negligible changes in the major backbone structures of $\mathrm{CNx}$.

Table 1. EA and BET surface area results for $\mathrm{CNx}$ and Cat-CNx.

\begin{tabular}{|c|c|c|}
\hline Materials & $\mathrm{CNx}$ & Cat-CNx \\
\hline $\mathrm{C}(\mathrm{wt}-\%)$ & 34.40 & 35.24 \\
\hline $\mathrm{N}(\mathrm{wt}-\%)$ & 59.21 & 55.35 \\
\hline C wt-\% divided by atomic weight & 2.86 & 2.93 \\
\hline N wt-\% divided by atomic weight & 4.22 & 3.95 \\
\hline C/N molar ratio & 0.68 & 0.74 \\
\hline Specific surface area $\left(\mathrm{m}^{2} \mathrm{~g}^{-1}\right)$ & 12.02 & 10.76 \\
\hline
\end{tabular}



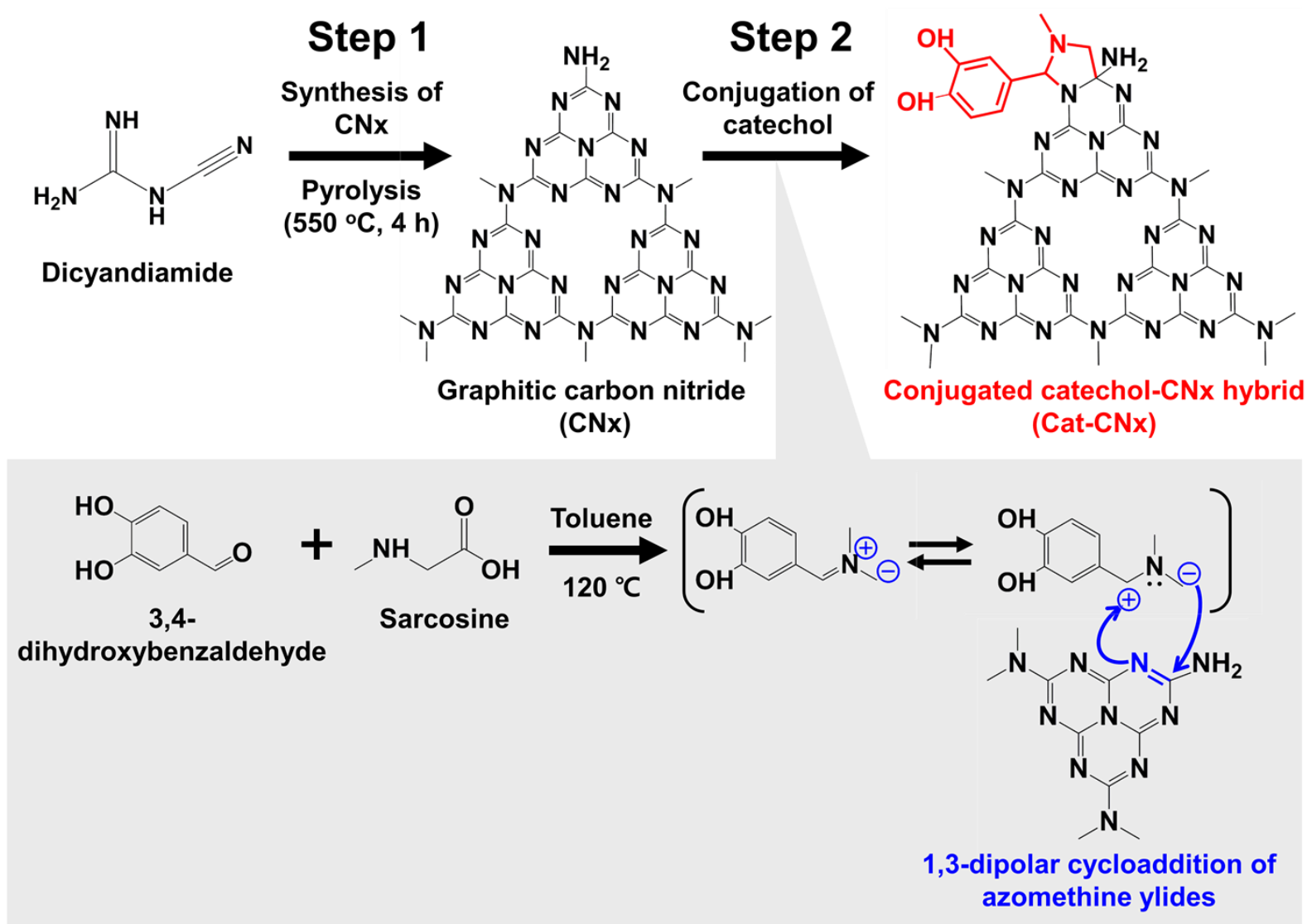

Scheme 1. Schematic illustration for the synthesis of $\mathrm{CNx}$ (step 1) and the subsequent conjugation of catechols to $\mathrm{CNx}$ using the 1,3-dipolar cycloaddition reaction (step 2). 


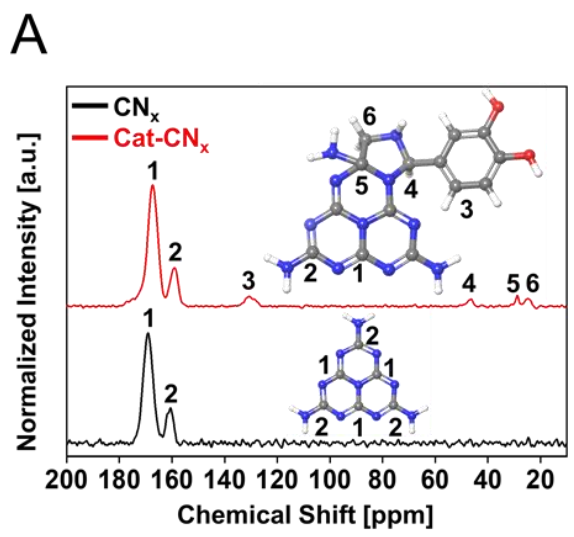

B
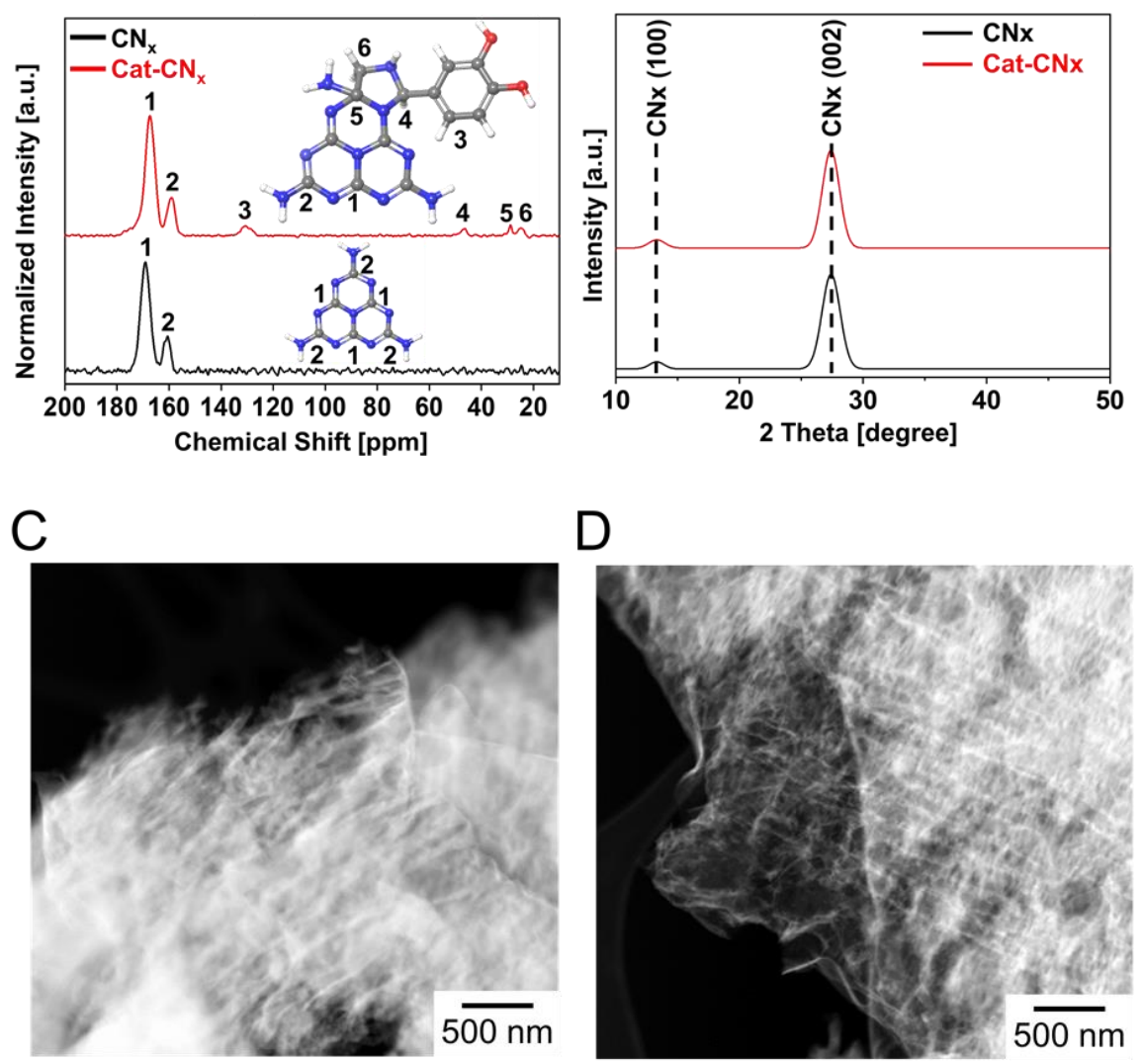

Figure 1. Solid-state ${ }^{13} \mathrm{C}$ CP-MAS-NMR (A) and XRD (B) spectra of CNx and Cat-CNx. STEM images of $\mathrm{CNx}(\mathrm{C})$ and Cat- $\mathrm{CNx}(\mathrm{D})$.

Electronic and Optical Characteristics. $\mathrm{CNx}$ exhibited a stark increment in absorption at a wavelength about $400 \mathrm{~nm}$, a typical absorption behavior of $\mathrm{CNx}$ as previously identified. ${ }^{50}$ Cat-CNx showed a broader absorption spectrum, covering almost all visible region, attributed to the enlarged delocalization of $\pi$-electrons caused by the introduction of a 5 -membered ring and catechol to $\mathrm{CNx}$ (Figure 2A). ${ }^{24}$ Tauc plot shows that the bandgap energy was reduced from 2.72 to $2.60 \mathrm{eV}$ after the catechol conjugation (inset of Figure 2A). X-ray photoelectron (XP) valence band spectra of $\mathrm{CNx}$ and Cat-CNx were analyzed for precise insight about electronic band 
structures by exploring valence band-edge positions (Figure 2B). ${ }^{9,39}$ The valence band edge was upshifted from 1.93 to $1.75 \mathrm{~V}$ (vs. NHE) after the catechol conjugation. Given the bandgap values obtained from the Tauc plot and the valence band edge position of both $\mathrm{CNx}$ and Cat-CNx, the conduction band edge also exhibited a slight upshift from -0.79 to -0.85 (vs. NHE). The valence and conduction band positions of $\mathrm{CNx}$ and Cat-CNx , determined by the UV-vis absorption and valence band-edge spectra, are summarized in Figure 2C.

X-ray photoelectron spectroscopy (XPS) was conducted to examine each element's chemical states and binding energies of each chemical bond in $\mathrm{CNx}$ and Cat-CNx. The $\mathrm{C}$ 1s spectrum of $\mathrm{CNx}$ exhibits a peak for heterocyclic component $(\mathrm{N}-\mathrm{C}=\mathrm{N})$ at $288.25 \mathrm{eV}$, while the peak was slightly upshifted to $288.55 \mathrm{eV}$ for Cat-CNx (Figure 2D and 2E). On the other hand, the binding energy of the $\mathrm{C}-\mathrm{O}$ peak in Cat-CNx was downshifted to $286.32 \mathrm{eV}$ with respect to the CO peak (286.45 eV) of 3,4-dihydroxybenzaldehyde, arising from C-OH of catechols (Figure 2E and 2F). The shifts of the binding energies for $\mathrm{N}-\mathrm{C}=\mathrm{N}$ and $\mathrm{C}-\mathrm{O}$ peaks indicate that the electron density was shifted from $\mathrm{CNx}$ to catechols because the binding energy of chemical bonds can be reduced by the shielding effect of electrons. ${ }^{47,51}$ The electron density polarization between $\mathrm{CNx}$ and catechols can induce an internal electric field. ${ }^{52}$ The electron density shift directionality can also be found in the N 1s spectra of $\mathrm{CNx}$ and Cat-CNx (Figure S7). The $\mathrm{C}=\mathrm{N}-\mathrm{C}$ peak's binding energy, originating from the $\pi$-conjugated systems of $\mathrm{CNx}$, upshifts from $398.85 \mathrm{eV}$ to $399.05 \mathrm{eV}$ when catechol was conjugated to $\mathrm{CNx}$. The electron density shift from $\mathrm{CNx}$ to catechols is consistent with previous reports on polydopamine-coated $\mathrm{CNx} \cdot{ }^{33,34}$ As a result, the upward bandbending can be induced at the surface of Cat-CNx, increasing the valence and conduction band edge positions and decreasing the electron density of the $\pi$-conjugated system at the surface of 
Cat-CNx compared to $\mathrm{CNx}$ as obtained from the XP valence band and XPS measurements for $\mathrm{C}$ 1s and N1s levels.

A
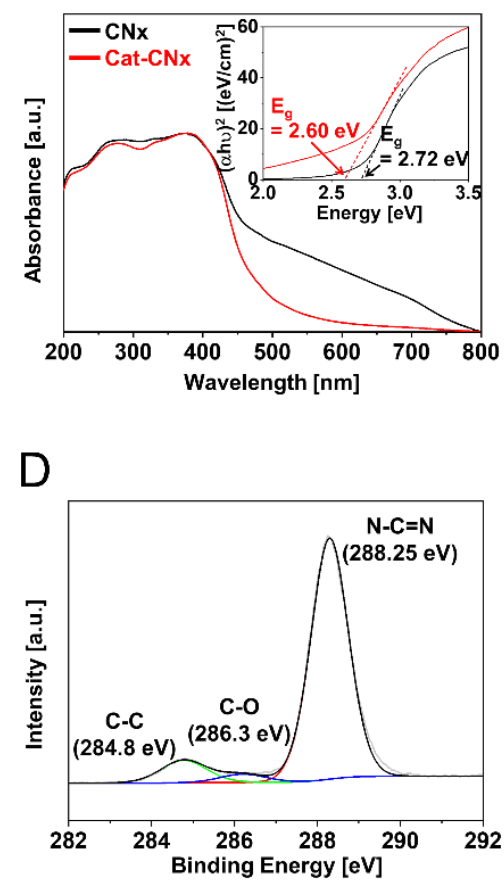

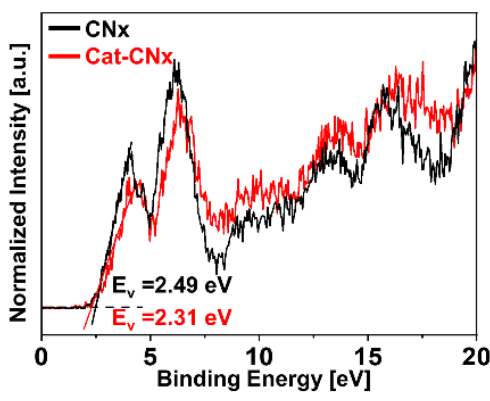

E

$\mathrm{B}$

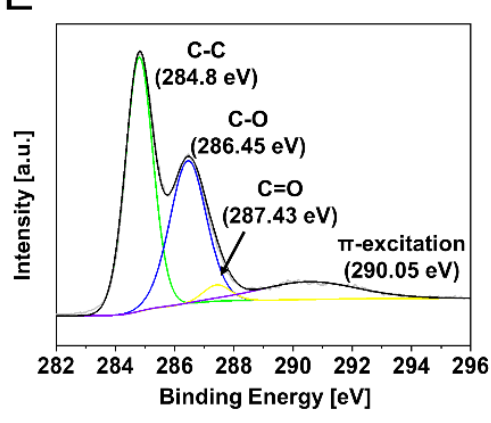

C

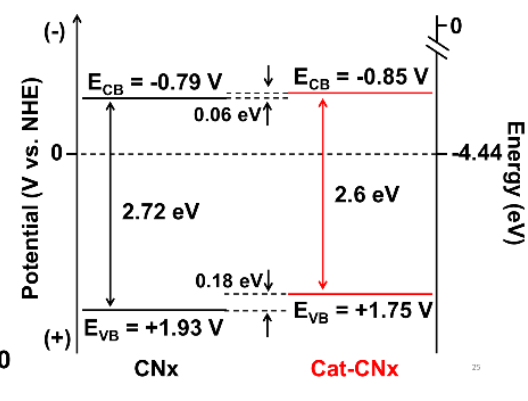

$\mathrm{F}$

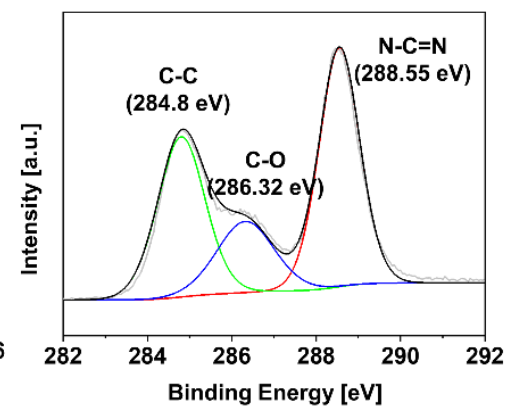

Figure 2. UV-vis absorption spectra (A, inset: Tauc plots), XP valence band spectra (B), and electronic structures (C) of $\mathrm{CNx}$ and Cat-CNx. XP $\mathrm{C} 1 \mathrm{~s}$ spectra of $\mathrm{CNx}$ (D), 3,4dihydroxybenzaldehyde (E), and Cat-CNx (F).

Behaviors of Photo-induced Charges. Since the possibility of electrons being attracted from $\mathrm{CNx}$ to catechols was found, it was assumed that the charge dynamics of Cat-CNx might be different from that of $\mathrm{CNx}$. To explore this assumption, we conducted the photoluminescence (PL) analysis of CNx and Cat-CNx with an excitation wavelength of $300 \mathrm{~nm}$ (Figure 3A). CNx showed a maximum PL intensity at $\sim 450 \mathrm{~nm}$ assigned to the recombination of photo-induced electronhole pairs by the band-to-band transition in the bulk phase of $\mathrm{CNx}$, as previously reported. ${ }^{53,54}$ Meanwhile, the conspicuous quenching of PL intensity $(\sim 94.5 \%)$ at $450 \mathrm{~nm}$ was observed for 
Cat-CNx with respect to $\mathrm{CNx}$, implying that the band-to-band recombination was significantly suppressed in Cat-CNx owing to the introduction of catechols. The hindered recombination can facilitate the separation of photo-induced charge carriers that can leads to enhanced photocatalytic reactions. ${ }^{55}$ Here, it is assumed that photo-induced electrons in the bulk phase of Cat-CNx might migrate to catechols, namely toward the edge of Cat-CNx, considering the electron density shifts observed in XPS.

To explore the charge dynamics inside Cat-CNx in detail, we conducted photoelectrochemical (PEC) analysis for photocurrent densities and open-circuit photovoltages using a three-electrode system at pH 7 under AM1.5-simulated illumination (denoted as '1 sun'). Fluorine-doped tin oxide (FTO) glass substrates were used to fabricate working electrodes coated with $\mathrm{CNx}$ and Cat-CNx. A bare FTO substrate exhibited negligible photoactivity in photocurrent and impedance measurements (Figure S8), implying that all the photo-responsive properties arose from the nature of $\mathrm{CNx}$ and Cat-CNx. A reduction reaction-operating condition ( $0.31 \mathrm{vs.} \mathrm{RHE)}$ after onset was used for photocurrent density measurements to trigger hydrogen evolution reaction from water with an overpotential of $0.1 \mathrm{~V}$ according to previous studies, determined by linear sweep voltammetry (LSV) (Figure 3B) ${ }^{56,57}$ Photocurrent densities were recorded for CNx and Cat-CNx under chopped light illumination at XXX mV vs. NHE, and the photocurrent density of Cat-CNx $\left(-0.752 \mu \mathrm{A} \mathrm{cm}^{-2}\right)$ was remarkably raised by 6.3 times compared to $\mathrm{CNx}\left(-0.120 \mu \mathrm{A} \mathrm{cm}{ }^{-2}\right)$ (Figure 3C). The increased photocurrent of Cat-CNx indicates that photoelectrons are transferred and utilized for reductive reactions more effectively compared to $\mathrm{CNx}$. In addition, open-circuit photovoltages were analyzed to examine the behaviors of photo-induced charges in $\mathrm{CNx}$ and Cat$\mathrm{CNx}$ in detail. While $\mathrm{CNx}$ showed a negative photovoltage of about $-14 \mathrm{mV}$, a typical n-type 
semiconductor's behavior, Cat-CNx exhibited a positive photovoltage of about $+25 \mathrm{mV}$ (Figure 3D). The positive photovoltage, generally found in p-type semiconductors, reflects that the majority of electrons migrate to the surface instead of drifting toward the bulk phase in typical ntype semiconductors due to upward band bending at the interface. ${ }^{9,55}$ The results suggest that the covalent attachment of catechol alters the direction of electrons' movement toward the opposite direction of CNx. Furthermore, the characteristic time of the photovoltage decay was analyzed to examine the efficiency of photo-induced charges in Cat-CNx and CNx (Figure S9 and Table S1, detailed analysis is provided in Supporting Information). The slower photovoltage decay of Cat$\mathrm{CNx}$ compared to $\mathrm{CNx}$ indicates more prolonged lifetime of photo-induced charges due to XXXXX. ${ }^{9}$ The facilitated photocatalytic behavior of Cat-CNx was also confirmed by the decrease in the charge transfer resistance at the semiconductor/electrolyte interface obtained from electrochemical impedance spectroscopy (EIS) analysis (Figure S10 and Table S2, detailed analysis is provided in Supporting Information).

Mott-Schottky analysis was conducted to clarify whether n-type characteristics of $\mathrm{CNx}$ were altered by the conjugation of catechols inducing the positive photovoltage (Figure 3E and 3F, detailed analysis provided in Supporting Information). CNx and Cat-CNx showed a positive slope determined by extrapolation in each linear region where the capacitance of the space charge region is far larger than that of the Helmholtz double layer. ${ }^{58}$ Since the slope is inversely proportional to a doping density, the positive slope of Cat-CNx indicates that Cat-CNx retains ntype characteristics of CNx. Furthermore, the flat-band potentials of $\mathrm{CNx}$ and Cat-CNx were determined by an $\mathrm{x}$-intercept of the extrapolation. Positive shifts of the flat-band potential by irradiation for both of $\mathrm{CNx}$ and Cat-CNx indicates smaller band-bending, namely smaller internal 
electric field compared to the dark. Note that the flat-band potential of Cat-CNx negatively shifted by about $-70 \mathrm{mV}$ with respect to $\mathrm{CNx}$ both in the dark and under illumination. The negative shift value of the flat-band potential was comparable to the degree of conduction band upshifts, implying that Fermi-level variation after the modification was negligible. The flat-band potential decrement indicates that the barrier height at the semiconductor/electrolyte interface for electron injection gets slightly higher primarily due to the upshift of conduction band edge of Cat-CNx compared to $\mathrm{CNx}$, leading to less thermodynamically favorable electrons' injection over the larger upward band-bending, i.e., larger electric field, for Cat-CNx than $\mathrm{CNx}$. Therefore, the increased photocurrent originates from the attraction of electrons toward the edge of Cat-CNx. The results suggest that Cat-CNx can provide a solution to overcome the existing issue of limited photochemical reductive performances of $\mathrm{CNx}$ due to its intrinsic n-type characteristics, as covered in a few previous reports. ${ }^{9,57,59-61}$

Recently, Bellamkonda et al. demonstrated that the introduction of aromatic rings to the repeating units of $\mathrm{CNx}$ generates additional intermediate states near the Fermi level and increases the charge separation efficiency. ${ }^{62}$ In addition, Kuriki et al. suggested two spatially separate energy states of $\mathrm{CNx}$ available for electrons to occupy under light illumination: main PL emissive states at the bulk that are intact by the existence of chemical species and non-emissive and reactive states at the edge ${ }^{53}$ Based on these results and our findings, we speculate that catechols' covalent attachment might produce additional intermediate states particularly at the edge to which redox couples are accessible. Furthermore, the conjugation might induce the perturbation in the equilibrium established between the populations of electrons excited to emissive states and those excited to non-emissive states. According to the positive photovoltage of Cat-CNx, electron- 
attracting properties of the catechol/quinone pair concentrate the electrons particularly at the surface intermediate states produced by catechols. The increased transition of electrons toward the surface states causes the decrement of the electrons' population at the main emissive states, evidenced by the large PL quenching. Furthermore, the larger internal electric field, as evidenced by the Mott-Schottky analysis, can sweep more photo-induced holes toward the edge to be consumed, contributing to the PL quenching. Furthermore, the introduction of the surface states extends the lifetime of photo-induced charges as evidenced by the slower photovoltage decay. ${ }^{62}$ Therefore, the higher photocurrent density of Cat-CNx results from the combined effect of the enhanced lifetime of photo-induced charges and the increased number of photo-induced electrons at the surface states accessible to redox species, where the surface states serve as a reactive site for photocatalysis. Based on these discussions, we summarize band energetics and reductive reaction processes of CNx and Cat-CNx under illumination schematically in Scheme 2. 
A

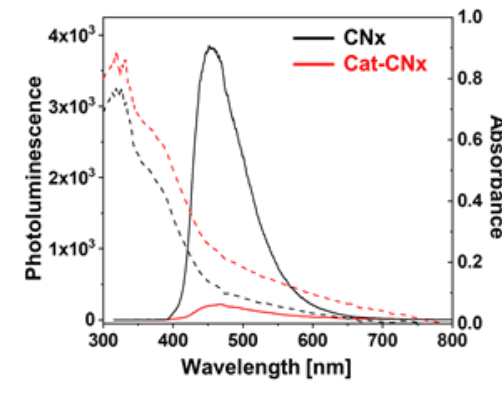

D

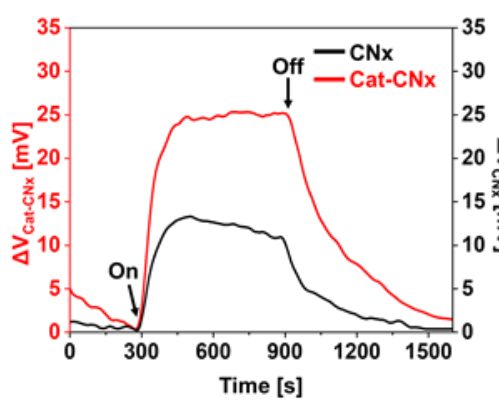

$\mathrm{B}$

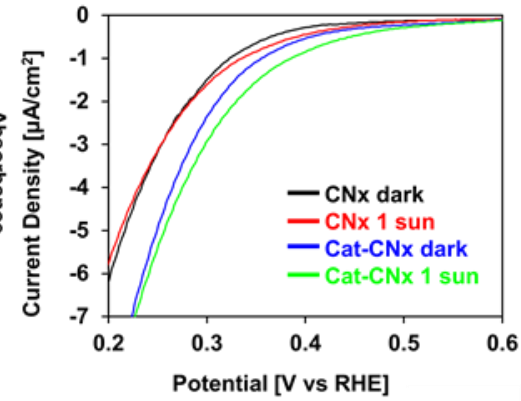

$E$

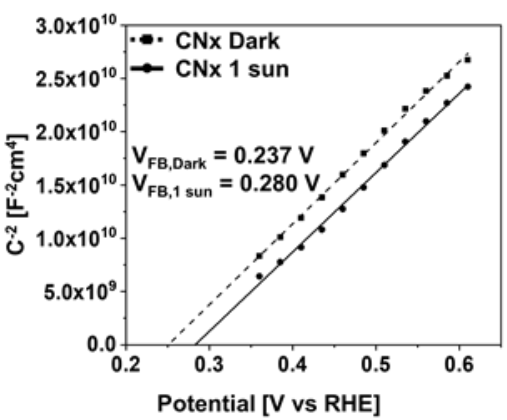

C

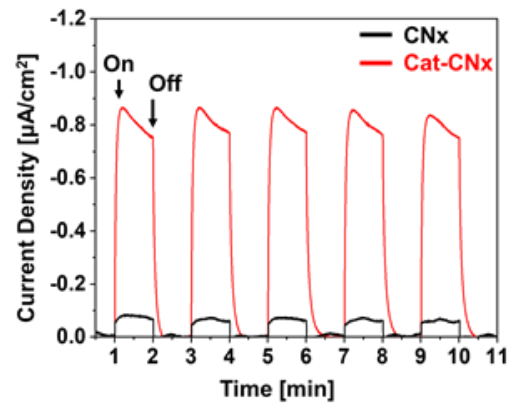

$\mathrm{F}$

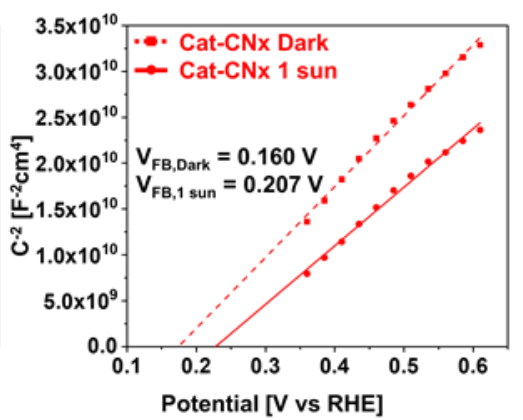

Figure 3. (A) PL (solid line) and UV-vis absorption (dashed line) spectra for dispersions of CNx and Cat-CNx at a concentration of $1 \mathrm{mg} \mathrm{mL}^{-1}$. (B) LSV curves for CNx and Cat-CNx in the dark and under illumination. (C) The photocurrent density of $\mathrm{CNx}$ and Cat-CNx at $0.31 \mathrm{~V}$ vs. RHE under chopped illumination. (D) Open-circuit photovoltage of $\mathrm{CNx}$ and Cat-CNx under chopped illumination. Mott-Schottky plots of $\mathrm{CNx}(\mathrm{E})$ and Cat-CNx (F).

A. Equilibrium in the dark

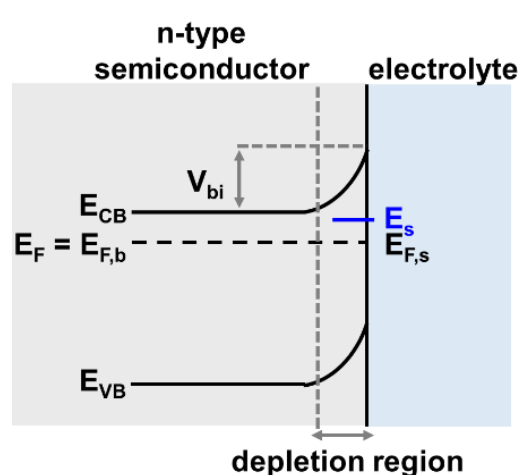

\section{B. CNx under illumination}

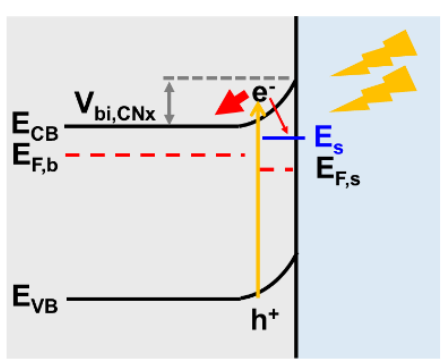

\section{Cat-CNx under illumination}

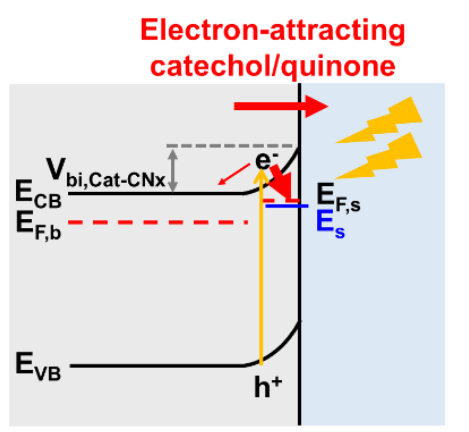

Scheme 2. Schematics of photo-enhanced reductive reaction processes with band energetics at the interface between $\mathrm{CNx}$ or Cat-CNx and electrolyte based on PEC measurements, where $\mathrm{E}_{\mathrm{CB}}=$ energy at the conduction band edge; $\mathrm{E}_{\mathrm{VB}}=$ energy at the velence band edge, $\mathrm{V}_{\mathrm{bi}}=$ built-in potential by the depletion region; $\mathrm{V}_{\mathrm{bi}, \mathrm{CNx}}=$ built-in potential of $\mathrm{CNx}$ under illumination; $\mathrm{V}_{\mathrm{bi}, \mathrm{Cat}-\mathrm{CNx}}=$ built- 
in potential of Cat-CNx under illumination; $\mathrm{E}_{\mathrm{F}}=$ Fermi level; $\mathrm{E}_{\mathrm{F}, \mathrm{b}}=$ electron quasi-Fermi level in the bulk; $E_{F, s}=$ electron quasi-Fermi level at the surface; $E_{\mathrm{s}}=$ surface states serving as a reactive site. (A) $\mathrm{CNx}$ or Cat-CNx as an n-type semiconductor at equilibrium in the dark, where $\mathrm{E}_{\mathrm{F}}=\mathrm{E}_{\mathrm{F}, \mathrm{b}}$ $=\mathrm{E}_{\mathrm{F}, \mathrm{s}}$ with $\mathrm{V}_{\mathrm{bi}}$. (B) $\mathrm{CNx}$ under illumination with a smaller $\mathrm{V}_{\mathrm{bi}, \mathrm{CNx}}$ than $\mathrm{V}_{\mathrm{bi}}$ where $\mathrm{E}_{\mathrm{F}, \mathrm{s}}$ lower than $\mathrm{E}_{\mathrm{F}, \mathrm{b}}$. In panel $\mathrm{B}$, photo-induced electrons move to the bulk rather than transfer to the surface states, resulting in the negative open-circuit photovoltage like the typical n-type semiconducting behaviors. (C) Cat-CNx under illumination with a smaller $\mathrm{V}_{\mathrm{bi}, \mathrm{Cat}-\mathrm{CNx}}$ than $\mathrm{V}_{\mathrm{bi}}$ where $\mathrm{E}_{\mathrm{F}, \mathrm{s}}$ higher than $\mathrm{E}_{\mathrm{F}, \mathrm{b}}$. In panel $\mathrm{C}$, photo-induced electrons move to the surface states rather than to the bulk in presence of conjugated catechols, resulting in the positive open-circuit photovoltage.

Photochemical Reduction of Gold Ions. Next, we demonstrated the applicability of the enhanced photo-enhanced reductive activity of Cat-CNx to noble metal ion recovery. Adsorption experiments for gold ions were conducted by dispersing $\mathrm{CNx}$ and Cat-CNx in gold ion solutions at various concentrations in the dark and under illumination. The number of adsorbed gold ions was calculated by determining the residual gold ion concentrations using inductively coupled plasma atomic emission spectroscopy (ICP-OES). Experimental data were applied to Langmuir and Freundlich isotherms to investigate the absorption behavior of gold ions (detailed isotherm analysis is described in the Supporting Information). For both $\mathrm{CNx}$ and Cat-CNx in the dark and under illumination, correlation coefficients obtained from the Langmuir isotherm were higher than those obtained from the Freundlich isotherm, indicating that gold ions were adsorbed on homogeneous surfaces and form a monolayer. ${ }^{29}$ The experimental results fitted to the Langmuir isotherm are shown in Figure 4A, and Langmuir isotherm parameters including the maximum gold absorption capacity obtained from the fitting were summarized in Table S4. In the dark, the maximum gold absorption capacity of Cat-CNx, XXXX, was raised by 2.9 times than that of $\mathrm{CNx}$, XXXX, due to the chelating and reducing capability of catechols for gold ions and a greater affinity for $\mathrm{AuCl}_{4}^{-}$ionic species induced by the functionalization of $\mathrm{CNx}$ with relatively hydrophilic catechol moieties. ${ }^{32}$ The higher standard reduction potential $\left(E^{0}\right)$ of quinone/catechol redox couple 
$\left(E^{0} \sim 0.8 \mathrm{~V}\right.$ vs. NHE) enables the reduction of $\mathrm{AuCl}_{4}^{-}$to $\mathrm{Au}^{0}$ with $E^{0}=+1.002 \mathrm{~V}$ (vs. NHE). Notably, the maximum gold absorption capacity of Cat-CNx under illumination, XXX, was raised by 4.3 times than that of $\mathrm{CNx}$, allowing an application of photochemically-assisted Cat-CNx to urban gold mining at a practical level requiring efficient gold ion recovery from electronic waste containing $\sim 200$ ppm of gold ions. ${ }^{63}$ Besides, the rate of increase in maximum gold adsorption capacity by irradiation was larger on Cat-CNx (1.94) than that on $\mathrm{CNx}(1.23)$. The results are attributed to the utilization of more photons due to the narrower bandgap energy of Cat-CNx and the efficient separation of photo-induced electron-hole pairs to be consumed. The reducing and hydrophilic properties of catechols can also contribute to the higher gold recovery efficiency. In addition, the standard Gibb's free energies of gold ion adsorption were derived from the Langmuir constants that represent how spontaneous the gold absorption reaction is (Table S6). ${ }^{64}$ The standard Gibb's free energies decreased by the presence of catechols and irradiation. Therefore, the synergetic effects of the increased affinity toward gold ions endowed by catechols and irradiation facilitate the gold ino absorption.

The gold-loaded $\mathrm{CNx}$ and Cat-CNx after the exposure to $1 \mathrm{mM}$ of gold ions are denoted as Au-CNx and Au-Cat-CNx, respectively. SEM, TEM, XRD, and XPS were used to identify the morphologies and chemical states of adsorbed $\mathrm{Au}$ with and without the catechol moieties and illumination. Gold nanoparticles (AuNPs) were found from Au-CNx and Au-Cat-CNx in SEM, TEM, and STEM images and identified by energy-dispersive X-ray spectroscopy (EDS) elemental mapping (Figure 4B, 4C, and S13-19). The size distributions of AuNPs were determined for at least 100 AuNPs observed by TEM images (Figure S20). The average diameter of AuNPs was increased by 3.1 times due to the innate chelating and reducing capability of catechols for gold 
ions by comparison between $\mathrm{Au}-\mathrm{CNx}$ and $\mathrm{Au}-\mathrm{Cat}-\mathrm{CNx}$ in the dark. Moreover, the average diameter of AuNPs from Au-Cat-CNx under illumination was raised by 1.6 times by contrast to the dark. The average diameter of AuNPs from $\mathrm{Au}-\mathrm{CNx}$ under illumination was increased by 1.3 times by comparison to the dark. The results indicate that photo-induced electrons are more efficiently utilized in Cat-CNx for reduction of gold ions and subsequent nucleation and growth of reduced gold atoms compared to $\mathrm{CNx}$. XRD patterns of $\mathrm{Au}-\mathrm{CNx}$ and $\mathrm{Au}-\mathrm{Cat}-\mathrm{CNx}$ showed peaks for metallic face-centered cubic gold at $2 \theta=38.2,44.4,64.6$, and 77.6 degrees, indicating the reduction of gold ions by catechol and photo-induced electrons except $\mathrm{Au}-\mathrm{CNx}$ in the dark (Figure 4D). Additionally, the characteristic peaks of $\mathrm{CNx}$ are still observed after the gold reduction, indicating that the exposure to gold ions did not induce remarkable distortions to the crystal structure of both CNx and Cat-CNx.

XPS analysis for $\mathrm{Au} 4 \mathrm{f}$ levels was conducted to investigate the chemical states of adsorbed gold. The deconvoluted XPS spectra and the atomic ratio of each chemical species show that more than 90 atomic percent of gold ions are reduced into their atomic state for all the cases of $\mathrm{CNx}$ and Cat-CNx in the dark and under illumination (Figure S21 and S22). The high ratio of the atomic state highlights the advantage of using $\mathrm{CNx}$ with the high conduction band edge compared to other semiconducting materials. ${ }^{37}$ Furthermore, based on the Langmuir isotherm and XPS analysis, the amount of gold absorbed on the surfaces depending on chemical states is showin in Figure 4E. The number of ionic states increases as catechols are introduced, corroborating that introduction of catechols increases the binding affinity to gold ions in aqueous media. Simultaneously, the organic texture of $\mathrm{CNx}$ impedes the tight binding with gold ions, making it easier for the ions to be washed away. Here, a clear increment in the amount of gold atomic state is observed by the 
introduction of catechols. Without light, Cat-CNx exhibited the 2.68 times increased amount of gold atomic state compared to $\mathrm{CNx}$. With exposure to light, while the amount of gold atomic state was raised by 1.23 times for $\mathrm{CNx}$, the amount increased up to 1.99 times for $\mathrm{Cat}-\mathrm{CNx}$, which implying the increased utilization of photo-induced charges.

Mechanisms of the photochemical reduction of gold ions on Cat-CNx particles are described in Figure 4F. Under light illumination, the photo-induced electrons are transferred to reduce $\mathrm{AuCl}_{4}^{-}$to $\mathrm{Au}^{0}$, while the photo-induced holes probably take part in the oxidation of water molecules and chloride anions generated from the reduction and dissociation of $\mathrm{AuCl}_{4}{ }^{-}$considering valence band maxima $(+1.75 \mathrm{~V})$ of Cat-CNx and standard reduction potentials of $\mathrm{O}_{2} / \mathrm{H}_{2} \mathrm{O}\left(E^{0}=+\right.$ $1.23 \mathrm{~V})$ and $\mathrm{Cl}_{2} / \mathrm{Cl}^{-}\left(E^{0}=+1.36 \mathrm{~V}\right)$ redox couples, as reported on photocatalytic hydrogen generation in seawater. ${ }^{65}$ 
A

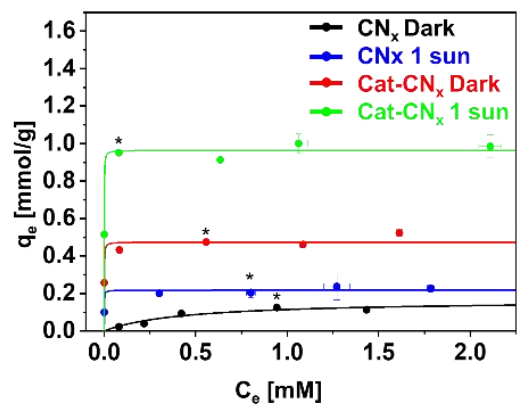

$\mathrm{D}$

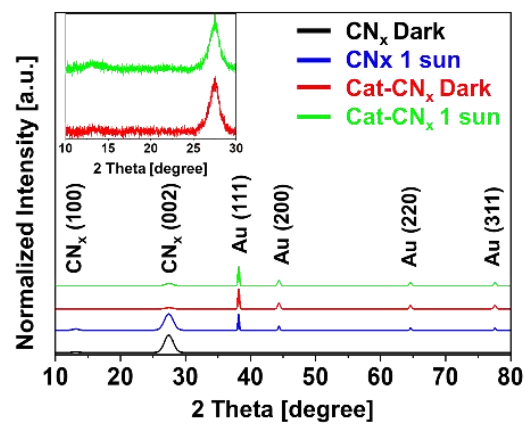

B

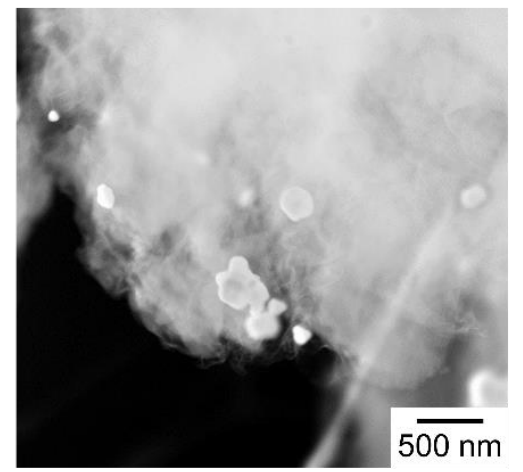

$\mathrm{E}$

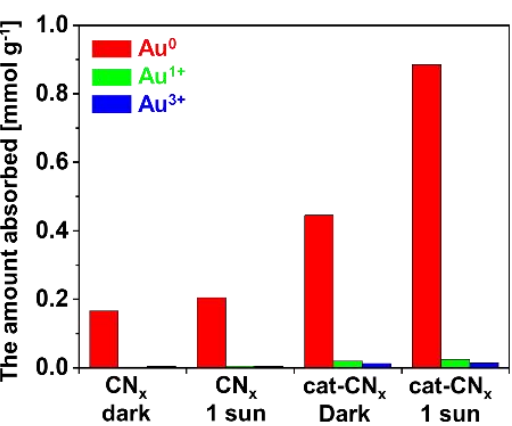

C

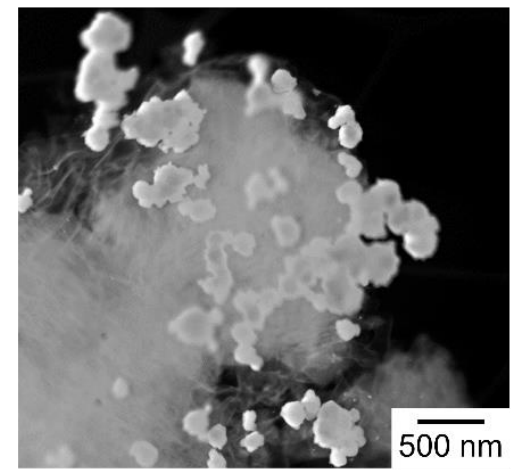

$\mathrm{F}$

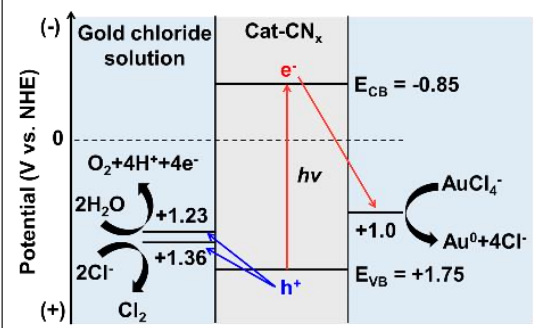

Figure 4. (A) Langmuir adsorption isotherm for gold ions. The asterisk marks denote the samples exposed to $1 \mathrm{mM}$ of gold ions under each condition. STEM images for $\mathrm{Au}-\mathrm{Cat}-\mathrm{CN}_{\mathrm{x}}$ without light (B) and with light (C). (D) XRD patterns for Au-CNx and Au-Cat-CNx. (E) The absorbed amount of gold depending on chemical states, (F) Mechanisms for photochemical reduction of gold ions by Cat-CNx in acidic aqueous solutions of gold ions.

\section{Conclusions}

We demonstrated that the chemical conjugation of catechols to the edge of $\mathrm{CNx}$ increased the photoreductive activity of $\mathrm{CNx}$ through the upshifts of band edges and polarization by the electron density shift from $\mathrm{CNx}$ to catechols. Importantly, owing to the catechol/quinone pairs' ability to attract electrons from $\mathrm{CNx}$, Cat-CNx exhibited positive open-circuit photovoltage opposite to pristine $\mathrm{CNx}$ showing a typical n-type behavior. Presumably, electrons are accumulated at the surface states induced by catechols rather than swept toward the bulk phase of $\mathrm{CNx}$. The results 
of photovoltage decay and PL quenching imply the prolonged lifetime and facilitated separation of photo-induced charges, leading favorable electron transfer from Cat-CNx to electrolytes. The photocurrent density of Cat-CNx was 6.3 times higher than that of $\mathrm{CNx}$, indicating the improved photocatalytic activity for reduction reactions, as supported by impedance analysis. Under light illumination, Cat-CNx exhibited 4.3 times higher maximum absorption capacity for gold ions compared to $\mathrm{CNx}$, allowing the practical application of Cat-CNx to urban gold mining. In this work we provide a new insight into the surface characteristics and photocatalytic activity engineered by the post-modification of $\mathrm{CNx}$.

\section{Experimental Methods}

Reagents. Dicyandiamide, 3,4-dihydroxybenzaldehyde, N-methylglycine (Sarcosine), toluene, gold(III) chloride trihydrate $\left(\mathrm{HAuCl}_{4} \cdot 3 \mathrm{H}_{2} \mathrm{O}\right)$, Nafion perfluorinated resin solution $(10 \mathrm{wt} \%$ in water), potassium phosphate dibasic trihydrate $\left(\mathrm{K}_{2} \mathrm{HPO}_{4} \cdot 3 \mathrm{H}_{2} \mathrm{O}\right)$, potassium phosphate monobasic $\left(\mathrm{KH}_{2} \mathrm{PO}_{4}\right)$, and sodium sulfate $\left(\mathrm{Na}_{2} \mathrm{SO}_{4}\right)$ were purchased from Sigma-Aldrich (St. Louis, MO, USA) and used without further purification.

Synthesis of CNx. CNx was synthesized by the simple pyrolysis of dicyandiamide. Four grams of dicyandiamide were placed on a porcelain boat and then heated with a tube furnace at $550{ }^{\circ} \mathrm{C}$ for $4 \mathrm{~h}$ with a ramp rate of $2.3{ }^{\circ} \mathrm{C}$ per min under an open ambient atmosphere. The yellow powder was carefully collected and ground with a mortar and pestle. Finally, the powder was washed with ethanol three times and deionized water twice and dried in a vacuum overnight.

Conjugation of Catechols to $\mathbf{C N x}$. Cat-CNx was synthesized according to a previously 
reported method ${ }^{24}$. Six hundred milligrams of $\mathrm{CNx}$ particles were dispersed in $300 \mathrm{~mL}$ toluene. Then, $4 \mathrm{mmol}(552.48 \mathrm{mg})$ of 3,4-dihydroxybenzaldehyde and $6 \mathrm{mmol}(534.54 \mathrm{mg})$ of $\mathrm{N}$ methylglycine were added to the dispersion. Next, the dispersion was heated at $120{ }^{\circ} \mathrm{C}$ for $24 \mathrm{~h}$ in an oil bath with vigorous stirring. After the reaction, the dispersion was centrifuged at $3000 \times \mathrm{g}$ for 3 min to isolate the products. The product was washed with ethanol three times and with deionized water twice and dried in vacuum overnight.

Fabrication of Electrodes. FTO-coated glass slides $(10 \mathrm{~mm} \times 15 \mathrm{~mm} \times 2.2 \mathrm{~mm}, 7 \mathrm{ohms})$ were used as a substrate to fabricate electrodes for photoelectrochemical measurements. The FTOcoated glass was cleaned using ultrasonic cleaning with acetone, ethanol, and deionized water and then dried. They were taped using a polyimide tape, saving an active area of $1 \mathrm{~cm}^{2}$. The $\mathrm{CNx}$ and Cat-CNx particles were dispersed in $5 \mathrm{wt} \%$ Nafion at a concentration of $10 \mathrm{mg} \mathrm{mL}^{-1}$ in a $1: 1$ mixture of ethanol and water and deposited on FTO-coated glass slides and then dried in vacuum at $70{ }^{\circ} \mathrm{C}$.

Photoelectrochemical Measurements. All photoelectrochemical measurements were performed in $0.1 \mathrm{M}$ potassium phosphate buffer $\left(\mathrm{KH}_{2} \mathrm{PO}_{4} / \mathrm{K}_{2} \mathrm{HPO}_{4}\right)$ with $0.1 \mathrm{M} \mathrm{Na}_{2} \mathrm{SO}_{4}$ at pH 7 using an electrochemical system (Ivium-n-Stat Multichannel electrochemical analyzer and IviumSoft) with a three-electrode system at room temperature. The FTO substrates loaded with $\mathrm{CNx}$ or Cat-CNx were used as a working electrode, and $\mathrm{Ag} / \mathrm{AgCl}$ and a platinum rod were used as reference and counter electrodes, respectively. Potentials against $\mathrm{Ag} / \mathrm{AgCl}$ were measured and converted to values against a reversible hydrogen electrode (RHE) using the following equation: $\mathrm{E}($ vs. $\mathrm{RHE})=\mathrm{E}(\mathrm{vs} . \mathrm{Ag} / \mathrm{AgCl})+0.197 \mathrm{~V}+0.059 \times \mathrm{pH}$. AM1.5-simulated illumination was applied to the working electrode using a solar simulator (ASAHI SPECTRA HAL-320) in all PEC 
measurements. Linear sweep voltammetry was carried out at a scan rate of $5 \mathrm{mV} \mathrm{s}^{-1}$ in the range of $0.11 \mathrm{~V}$ to $2.11 \mathrm{~V}$ (vs. RHE). Mott-Schottky plots were obtained with a scan range of $0.11 \mathrm{~V}$ to $2.11 \mathrm{~V}$ (vs. RHE) at 500, 1000, and $2000 \mathrm{~Hz}$ of frequencies. Electrochemical impedance spectroscopy was conducted at $0.31 \mathrm{~V}$ (vs. RHE) with $0.01 \mathrm{~V}$ amplitude in the frequency range of 10000-0.1 Hz. Photocurrent densities were measured by chronoamperometry under chopped illumination, and the light was switched on and off at an interval of $60 \mathrm{~s}$ at $0.31 \mathrm{~V}$ (vs. RHE). Photovoltages were analyzed by measuring open-circuit voltage. The working electrodes were immersed in the dark until the systems reached their equilibrium. After reaching the equilibrium, the working electrodes were exposed to light until the photovoltage became stable. When the variation of photovoltage became negligible, light was turned off and the decaying of photovoltage was monitored.

Gold Adsorption Isotherm. A gold isotherm experiment was conducted by following a previously reported method with slight modification ${ }^{29}$. In the case of $\mathrm{CNx}, 4 \mathrm{mg}$ of $\mathrm{CNx}$ particles were put in a $4 \mathrm{~mL}$ glass vial. In the case of Cat-CNx, $0.4 \mathrm{~mL}$ of Cat-CNx aqueous dispersion, having a concentration of $10 \mathrm{mg} \mathrm{mL}^{-1}$ was added in a $4 \mathrm{~mL}$ glass vial. The difference originates from different dispersion stability of two particles in an aqueous solution. The designated amount of deionized water and a $10 \mathrm{mM}$ of $\mathrm{HAuCl}_{4}$ aqueous solution were added to make $4 \mathrm{~mL}$ of total volume and adjust the particle concentration with $1 \mathrm{mg} \mathrm{mL}^{-1}$, and gold ion concentration from 0.1 $\mathrm{mM}$ up to $3 \mathrm{mM}$ depending on the conditions applied. The dispersions were stirred in the dark and 1-sun condition at room temperature for $3 \mathrm{~h}$. After the reaction, the $\mathrm{CNx}$ particles were collected by centrifugation at $5000 \times \mathrm{g}$ for $3 \mathrm{~min}$, and the supernatant was filtered using syringe filters with a pore diameter of $0.2 \mu \mathrm{m}$. The filtered supernatants were diluted 10 times (in terms of weight) 
with deionized water to measure the gold ion concentration with inductively coupled plasma atomic emission spectroscopy (ICP-OES). The collected particles were washed with deionized water twice and then ethanol, and finally dried in vacuum.

Characterization. Nitrogen sorption analysis was performed using a 3Flex Surface Characterization Analyzer (Micromeritics Corp., Norcross, GA, USA) at $77 \mathrm{~K}$. Before the analysis, samples were degassed at $150^{\circ} \mathrm{C}$ for $24 \mathrm{~h}$. Fourier Transform Infrared (FT-IR, Jasco FT/IR-6100, Tokyo, Japan) spectroscopy was conducted to identify the chemical modifications of CNx using a standard $\mathrm{KBr}$ pellet method. Solid-state ${ }^{13} \mathrm{C}$ cross-polarization magic angle spinning nuclear magnetic resonance $\left({ }^{13} \mathrm{C}\right.$ CP-MAS NMR) was conducted at room temperature to verify the introduction through Agilent 400 MHz 54 mm NMR DD2 (Agilent Technologies, Santa Clara, CA, USA). The crystal structures of $\mathrm{CNx}$, Cat-CNx, and adsorbed gold were determined by X-ray diffraction (XRD, Rigaku Smartlab, Tokyo, Japan) with $\mathrm{Cu}$ K $\alpha$ radiation $(\lambda=0.154 \mathrm{~nm})$. Elemental Analysis (Thermo Fisher Scientific FlashSmart, Waltham, MA, USA) was performed to characterize the $\mathrm{C} / \mathrm{N}$ ratio of the samples. X-ray photoelectron spectroscopy (XPS, KRATOS AxisSupra, Kyoto, Japan) was obtained to explore valence bands and chemical states of CNx, Cat-CNx, Au-CNx, and Au-Cat-CNx using a monochromatized Al Ka (1486.7 eV) X-ray source. Photoluminescence spectra were obtained using a fluorescence spectrophotometer (Hitachi F-7000, Tokyo, Japan). In detail, the dispersions containing $\mathrm{CNx}$ and Cat-CNx at a concentration of $1 \mathrm{mg}$ $\mathrm{mL}^{-1}$ were excited by laser with a wavelength of $300 \mathrm{~nm}$, and the wavelength of emitted light was monitored from $315 \mathrm{~nm}$ to $800 \mathrm{~nm}$. Field emission transmission electron microscopy (TEM, FEI Company Tecnai F20, Hillsboro, Oregon, USA) was used with an accelerating voltage of $300 \mathrm{kV}$ to analyze the size and morphologies of $\mathrm{CNx}$, Cat-CNx, $\mathrm{Au}-\mathrm{CNx}$, and Au- Cat-CNx. Inductively 
coupled plasma-optical emission spectroscopy (ICP-OES, Agilent Technologies, Santa Clara, CA, USA) was used to measure the concentration of residual gold ions to quantify the amount of gold absorbed on both $\mathrm{CNx}$ and Cat-CNx.

\section{ASSOCIATED CONTENT \\ Supporting Information.}

\section{AUTHOR INFORMATION \\ Corresponding Author}

\section{ORCID}

\section{Author Contributions}

\section{ACKNOWLEDGMENT}

\section{REFERENCES}

(1) Wang, Y.; Wang, X.; Antonietti, M. Polymeric Graphitic Carbon Nitride as a Heterogeneous Organocatalyst: From Photochemistry to Multipurpose Catalysis to Sustainable Chemistry. Angew. Chemie - Int. Ed. 2012, 51 (1), 68-89. https://doi.org/10.1002/anie.201101182.

(2) Huda, M. N.; Turner, J. A. Morphology-Dependent Optical Absorption and Conduction Properties of Photoelectrochemical Photocatalysts for H2 Production: A Case Study. J. Appl. Phys. 2010, 107 (12). https://doi.org/10.1063/1.3428957.

(3) Safaei, J.; Mohamed, N. A.; Mohamad Noh, M. F.; Soh, M. F.; Ludin, N. A.; Ibrahim, M. A.; Roslam Wan Isahak, W. N.; Mat Teridi, M. A. Graphitic Carbon Nitride (g-C3N4) Electrodes for Energy Conversion and Storage: A Review on Photoelectrochemical Water Splitting, Solar Cells and Supercapacitors. Journal of Materials Chemistry A. Royal Society of Chemistry 2018, pp 22346-22380. https://doi.org/10.1039/c8ta08001a.

(4) Wang, X.; Maeda, K.; Thomas, A.; Takanabe, K.; Xin, G.; Carlsson, J. M.; Domen, K.; Antonietti, M. A Metal-Free Polymeric Photocatalyst for Hydrogen Production from Water under Visible Light. Nat. Mater. 2009, 8 (1), 76-80. https://doi.org/10.1038/nmat2317.

(5) Niu, W.; Yang, Y. Graphitic Carbon Nitride for Electrochemical Energy Conversion and Storage. ACS Energy Lett. 2018, 3 (11), 2796-2815. https://doi.org/10.1021/acsenergylett.8b01594.

(6) Talapaneni, S. N.; Singh, G.; Kim, I. Y.; AlBahily, K.; Al-Muhtaseb, A. H.; Karakoti, A. S.; Tavakkoli, E.; Vinu, A. Nanostructured Carbon Nitrides for CO2 Capture and 
Conversion. Adv. Mater. 2020, 32 (18), 1-21. https://doi.org/10.1002/adma.201904635.

Zhao, G.; Huang, X.; Wang, X.; Wang, X. Progress in Catalyst Exploration for Heterogeneous CO2 Reduction and Utilization: A Critical Review. J. Mater. Chem. A 2017, 5 (41), 21625-21649. https://doi.org/10.1039/c7ta07290b.

(8) Hong, Z.; Shen, B.; Chen, Y.; Lin, B.; Gao, B. Enhancement of Photocatalytic H2 Evolution over Nitrogen-Deficient Graphitic Carbon Nitride. J. Mater. Chem. A 2013, 1 (38), 11754-11761. https://doi.org/10.1039/c3ta12332d.

(9) Ruan, Q.; Miao, T.; Wang, H.; Tang, J. Insight on Shallow Trap States-Introduced Photocathodic Performance in n-Type Polymer Photocatalysts. J. Am. Chem. Soc. 2020, 142 (6), 2795-2802. https://doi.org/10.1021/jacs.9b10476.

(10) Hutton, G. A. M.; Martindale, B. C. M.; Reisner, E. Carbon Dots as Photosensitisers for Solar-Driven Catalysis. Chem. Soc. Rev. 2017, 46 (20), 6111-6123. https://doi.org/10.1039/c7cs00235a.

(11) Niu, P.; Yin, L. C.; Yang, Y. Q.; Liu, G.; Cheng, H. M. Increasing the Visible Light Absorption of Graphitic Carbon Nitride (Melon) Photocatalysts by Homogeneous SelfModifi Cation with Nitrogen Vacancies. Adv. Mater. 2014, 26 (47), 8046-8052. https://doi.org/10.1002/adma.201404057.

(12) Shevlin, S. A.; Guo, Z. X. Anionic Dopants for Improved Optical Absorption and Enhanced Photocatalytic Hydrogen Production in Graphitic Carbon Nitride. Chem. Mater. 2016, 28 (20), 7250-7256. https://doi.org/10.1021/acs.chemmater.6b02002.

(13) Yu, H.; Shi, R.; Zhao, Y.; Bian, T.; Zhao, Y.; Zhou, C.; Waterhouse, G. I. N.; Wu, L. Z.; Tung, C. H.; Zhang, T. Alkali-Assisted Synthesis of Nitrogen Deficient Graphitic Carbon Nitride with Tunable Band Structures for Efficient Visible-Light-Driven Hydrogen Evolution. Adv. Mater. 2017, 29 (16), 1-7. https://doi.org/10.1002/adma.201605148.

(14) Liu, G.; Niu, P.; Sun, C.; Smith, S. C.; Chen, Z.; Lu, G. Q.; Cheng, H. M. Unique Electronic Structure Induced High Photoreactivity of Sulfur-Doped Graphitic C3N4. J. Am. Chem. Soc. 2010, 132 (33), 11642-11648. https://doi.org/10.1021/ja103798k.

(15) Yue, B.; Li, Q.; Iwai, H.; Kako, T.; Ye, J. Hydrogen Production Using Zinc-Doped Carbon Nitride Catalyst Irradiated with Visible Light. Sci. Technol. Adv. Mater. 2011, 12 (3). https://doi.org/10.1088/1468-6996/12/3/034401.

(16) Zhang, Y.; Mori, T.; Ye, J.; Antonietti, M. Phosphorus-Doped Carbon Nitride Solid: Enhanced Electrical Conductivity and Photocurrent Generation. J. Am. Chem. Soc. 2010, 132 (18), 6294-6295. https://doi.org/10.1021/ja101749y.

(17) Wang, Y.; Li, H.; Yao, J.; Wang, X.; Antonietti, M. Synthesis of Boron Doped Polymeric Carbon Nitride Solids and Their Use as Metal-Free Catalysts for Aliphatic C-H Bond Oxidation. Chem. Sci. 2011, 2 (3), 446-450. https://doi.org/10.1039/c0sc00475h.

(18) Wang, Y.; Zhang, J.; Wang, X.; Antonietti, M.; Li, H. Boron- and Fluorine-Containing Mesoporous Carbon Nitride Polymers: Metal-Free Catalysts for Cyclohexane Oxidation. Angew. Chemie - Int. Ed. 2010, 49 (19), 3356-3359.

https://doi.org/10.1002/anie.201000120.

(19) Hong, S.; Kim, J.; Na, Y. S.; Park, J.; Kim, S.; Singha, K.; Im, G. Il; Han, D. K.; Kim, W. J.; Lee, H. Poly(Norepinephrine): Ultrasmooth Material-Independent Surface Chemistry and Nanodepot for Nitric Oxide. Angew. Chemie - Int. Ed. 2013, 52 (35), 9187-9191. https://doi.org/10.1002/anie.201301646.

(20) Guo, Y.; Yang, J.; Chu, S.; Kong, F.; Luo, L.; Wang, Y.; Zou, Z. Theoretical and 
Experimental Study on Narrowing the Band Gap of Carbon Nitride Photocatalyst by Coupling a Wide Gap Molecule. Chem. Phys. Lett. 2012, 550, 175-180.

https://doi.org/10.1016/j.cplett.2012.09.019.

(21) Dutta, R.; Dey, B.; Kalita, D. J. Narrowing the Band Gap of Graphitic Carbon Nitride Sheet by Coupling Organic Moieties: A DFT Approach. Chem. Phys. Lett. 2018, 707, 101-107. https://doi.org/10.1016/j.cplett.2018.07.038.

(22) Huang, Z.; Chen, H.; Zhao, L.; Fang, W.; He, X.; Li, W.; Tian, P. In Suit Inducing Electron-Donating and Electron-Withdrawing Groups in Carbon Nitride by One-Step NH4Cl-Assisted Route: A Strategy for High Solar Hydrogen Production Efficiency. Environ. Int. 2019, 126 (October 2018), 289-297. https://doi.org/10.1016/j.envint.2019.02.030.

(23) Chu, S.; Wang, Y.; Guo, Y.; Feng, J.; Wang, C.; Luo, W.; Fan, X.; Zou, Z. Band Structure Engineering of Carbon Nitride: In Search of a Polymer Photocatalyst with High Photooxidation Property. ACS Catal. 2013, 3 (5), 912-919. https://doi.org/10.1021/cs4000624.

(24) Zhang, P.; Li, H.; Wang, Y. Post-Functionalization of Graphitic Carbon Nitrides by Grafting Organic Molecules: Toward C-H Bond Oxidation Using Atmospheric Oxygen. Chem. Commun. 2014, 50 (48), 6312-6315. https://doi.org/10.1039/c4cc02676d.

(25) Tian, J.; Zhang, L.; Fan, X.; Zhou, Y.; Wang, M.; Cheng, R.; Li, M.; Kan, X.; Jin, X.; Liu, Z.; et al. A Post-Grafting Strategy to Modify g-C3N4 with Aromatic Heterocycles for Enhanced Photocatalytic Activity. J. Mater. Chem. A 2016, 4 (36), 13814-13821. https://doi.org/10.1039/c6ta04297j.

(26) Vidyasagar, D.; Ghugal, S. G.; Umare, S. S.; Banavoth, M. Extended $\pi$-Conjugative n-p Type Homostructural Graphitic Carbon Nitride for Photodegradation and Charge-Storage Applications. Sci. Rep. 2019, 9 (1), 1-10. https://doi.org/10.1038/s41598-019-43312-5.

(27) Lee, H.; Dellatore, S. M.; Miller, W. M.; Messersmith, P. B. Mussel-Inspired Surface Chemistry for Multifunctional Coatings. Science (80-. ). 2007, 318 (5849), 426-430. https://doi.org/10.1126/science.1147241.

(28) Kim, I.; Son, H. Y.; Yang, M. Y.; Nam, Y. S. Bioinspired Design of an Immobilization Interface for Highly Stable, Recyclable Nanosized Catalysts. ACS Appl. Mater. Interfaces 2015, 7 (26), 14415-14422. https://doi.org/10.1021/acsami.5b03249.

(29) Kim, J.; Kim, K. R.; Hong, Y.; Choi, S.; Yavuz, C. T.; Kim, J. W.; Nam, Y. S. Photochemically Enhanced Selective Adsorption of Gold Ions on Tannin-Coated Porous Polymer Microspheres. ACS Appl. Mater. Interfaces 2019, 11 (24), 21915-21925. https://doi.org/10.1021/acsami.9b05197.

(30) Kim, Y. J.; Wu, W.; Chun, S. E.; Whitacre, J. F.; Bettinger, C. J. Catechol-Mediated Reversible Binding of Multivalent Cations in Eumelanin Half-Cells. Adv. Mater. 2014, 26 (38), 6572-6579. https://doi.org/10.1002/adma.201402295.

(31) Song, Y.; Jiang, H.; Wang, B.; Kong, Y.; Chen, J. Silver-Incorporated Mussel-Inspired Polydopamine Coatings on Mesoporous Silica as an Efficient Nanocatalyst and Antimicrobial Agent. ACS Appl. Mater. Interfaces 2018, 10 (2), 1792-1801. https://doi.org/10.1021/acsami.7b18136.

(32) Saiz-Poseu, J.; Mancebo-Aracil, J.; Nador, F.; Busqué, F.; Ruiz-Molina, D. The Chemistry behind Catechol-Based Adhesion. Angew. Chemie - Int. Ed. 2019, 58 (3), 696-714. https://doi.org/10.1002/anie.201801063. 
(33) Cai, J.; Huang, J.; Wang, S.; Iocozzia, J.; Sun, Z.; Sun, J.; Yang, Y.; Lai, Y.; Lin, Z. Crafting Mussel-Inspired Metal Nanoparticle-Decorated Ultrathin Graphitic Carbon Nitride for the Degradation of Chemical Pollutants and Production of Chemical Resources. Adv. Mater. 2019, 31 (15), 1806314. https://doi.org/10.1002/adma.201806314.

(34) Yu, Z.; Li, F.; Yang, Q.; Shi, H.; Chen, Q.; Xu, M. Nature-Mimic Method to Fabricate Polydopamine/Graphitic Carbon Nitride for Enhancing Photocatalytic Degradation Performance. ACS Sustain. Chem. Eng. 2017, 5 (9), 7840-7850. https://doi.org/10.1021/acssuschemeng.7b01313.

(35) Yang, W.; Godin, R.; Kasap, H.; Moss, B.; Dong, Y.; Hillman, S. A. J.; Steier, L.; Reisner, E.; Durrant, J. R. Electron Accumulation Induces Efficiency Bottleneck for Hydrogen Production in Carbon Nitride Photocatalysts. J. Am. Chem. Soc. 2019, 141 (28), 11219-11229. https://doi.org/10.1021/jacs.9b04556.

(36) Cho, S.; Kim, S. H. Hydroxide Ion-Mediated Synthesis of Monodisperse DopamineMelanin Nanospheres. J. Colloid Interface Sci. 2015, 458, 87-93. https://doi.org/10.1016/j.jcis.2015.06.051.

(37) Kim, K. R.; Choi, S.; Yavuz, C. T.; Nam, Y. S. Direct Z-Scheme Tannin-TiO2 Heterostructure for Photocatalytic Gold Ion Recovery from Electronic Waste. ACS Sustain. Chem. Eng. 2020. https://doi.org/10.1021/acssuschemeng.0c00860.

(38) Fina, F.; Callear, S. K.; Carins, G. M.; Irvine, J. T. S. Structural Investigation of Graphitic Carbon Nitride via XRD and Neutron Diffraction. Chem. Mater. 2015, 27 (7), 2612-2618. https://doi.org/10.1021/acs.chemmater.5b00411.

(39) Zhao, D.; Dong, C. L.; Wang, B.; Chen, C.; Huang, Y. C.; Diao, Z.; Li, S.; Guo, L.; Shen, S. Synergy of Dopants and Defects in Graphitic Carbon Nitride with Exceptionally Modulated Band Structures for Efficient Photocatalytic Oxygen Evolution. Adv. Mater. 2019, 31 (43), 1903545. https://doi.org/10.1002/adma.201903545.

(40) Finkelstein-Shapiro, D.; Fournier, M.; Méndez-Hernández, D. D.; Guo, C.; Calatayud, M.; Moore, T. A.; Moore, A. L.; Gust, D.; Yarger, J. L. Understanding Iridium Oxide Nanoparticle Surface Sites by Their Interaction with Catechol. Phys. Chem. Chem. Phys. 2017, 19 (24), 16151-16158. https://doi.org/10.1039/c7cp01516j.

(41) Finkelstein-Shapiro, D.; Davidowski, S. K.; Lee, P. B.; Guo, C.; Holland, G. P.; Rajh, T.; Gray, K. A.; Yarger, J. L.; Calatayud, M. Direct Evidence of Chelated Geometry of Catechol on TiO2 by a Combined Solid-State NMR and DFT Study. J. Phys. Chem. C 2016, 120 (41), 23625-23630. https://doi.org/10.1021/acs.jpcc.6b08041.

(42) Baccile, N.; Falco, C.; Titirici, M. M. Characterization of Biomass and Its Derived Char Using 13C-Solid State Nuclear Magnetic Resonance. Green Chemistry. Royal Society of Chemistry December 2014, pp 4839-4869. https://doi.org/10.1039/c3gc42570c.

(43) Soares-Santos, P. C. R.; Trindade, T.; Tomé, A. C.; Rocha, J.; Sá Ferreira, R. A.; Carlos, L. D.; Almeida Paz, F. A.; Klinowski, J.; Nogueira, H. I. S. Lanthanide Compounds Containing a Benzo-15-Crown-5 Derivatised [60]Fullerene and the Related [Tb(H2O)3(NO 3)2(Acac)] • C14H20O 5 Supramolecular Adduct. New J. Chem. 2004, 28 (11), 1352-1358. https://doi.org/10.1039/b410068a.

(44) Botari, T.; Huhn, W. P.; Lau, V. W. H.; Lotsch, B. V.; Blum, V. Thermodynamic Equilibria in Carbon Nitride Photocatalyst Materials and Conditions for the Existence of Graphitic Carbon Nitride G-C3N4. Chem. Mater. 2017, 29 (10), 4445-4453. https://doi.org/10.1021/acs.chemmater.7b00965. 
(45) Barrett, D. G.; Sileika, T. S.; Messersmith, P. B. Molecular Diversity in Phenolic and Polyphenolic Precursors of Tannin-Inspired Nanocoatings. Chem. Commun. 2014, 50 (55), 7265-7268. https://doi.org/10.1039/c4cc02961e.

(46) Chatterjee, S.; Prados-Rosales, R.; Tan, S.; Itin, B.; Casadevall, A.; Stark, R. E. Demonstration of a Common Indole-Based Aromatic Core in Natural and Synthetic Eumelanins by Solid-State NMR. Org. Biomol. Chem. 2014, 12 (34), 6730-6736. https://doi.org/10.1039/c4ob01066c.

(47) Uekert, T.; Kasap, H.; Reisner, E. Photoreforming of Nonrecyclable Plastic Waste over a Carbon Nitride/Nickel Phosphide Catalyst. J. Am. Chem. Soc. 2019, 141 (38), 1520115210. https://doi.org/10.1021/jacs.9b06872.

(48) Liao, Y.; Zhu, S.; Chen, Z.; Lou, X.; Zhang, D. A Facile Method of Activating Graphitic Carbon Nitride for Enhanced Photocatalytic Activity. Phys. Chem. Chem. Phys. 2015, 17 (41), 27826-27832. https://doi.org/10.1039/c5cp05186j.

(49) Liu, J.; Liu, Y.; Liu, N.; Han, Y.; Zhang, X.; Huang, H.; Lifshitz, Y.; Lee, S. T.; Zhong, J.; Kang, Z. Metal-Free Efficient Photocatalyst for Stable Visible Water Splitting via a TwoElectron Pathway. Science (80-. ). 2015, 347 (6225), 970-974. https://doi.org/10.1126/science.aaa3145.

(50) Zhang, G.; Zhang, M.; Ye, X.; Qiu, X.; Lin, S.; Wang, X. Iodine Modified Carbon Nitride Semiconductors as Visible Light Photocatalysts for Hydrogen Evolution. Adv. Mater. 2014, 26 (5), 805-809. https://doi.org/10.1002/adma.201303611.

(51) Lau, V. W. H.; Moudrakovski, I.; Botari, T.; Weinberger, S.; Mesch, M. B.; Duppel, V.; Senker, J.; Blum, V.; Lotsch, B. V. Rational Design of Carbon Nitride Photocatalysts by Identification of Cyanamide Defects as Catalytically Relevant Sites. Nat. Commun. 2016, 7. https://doi.org/10.1038/ncomms12165.

(52) Gurrentz, J. M.; Rose, M. J. Non-Catalytic Benefits of Ni(II) Binding to an Si(111)-PNP Construct for Photoelectrochemical Hydrogen Evolution Reaction: Metal Ion Induced Flat Band Potential Modulation. J. Am. Chem. Soc. 2020, 142 (12), 5657-5667. https://doi.org/10.1021/jacs.9b12824.

(53) Kuriki, R.; Ranasinghe, C. S. K.; Yamazaki, Y.; Yamakata, A.; Ishitani, O.; Maeda, K. Excited-State Dynamics of Graphitic Carbon Nitride Photocatalyst and Ultrafast Electron Injection to a $\mathrm{Ru}(\mathrm{II})$ Mononuclear Complex for Carbon Dioxide Reduction. J. Phys. Chem. C 2018, 122 (29), 16795-16802. https://doi.org/10.1021/acs.jpcc.8b03996.

(54) Ma, B.; Chen, G.; Fave, C.; Chen, L.; Kuriki, R.; Maeda, K.; Ishitani, O.; Lau, T. C.; Bonin, J.; Robert, M. Efficient Visible-Light-Driven CO2 Reduction by a Cobalt Molecular Catalyst Covalently Linked to Mesoporous Carbon Nitride. J. Am. Chem. Soc. 2020, 142 (13), 6188-6195. https://doi.org/10.1021/jacs.9b13930.

(55) Zhao, D.; Dong, C. L.; Wang, B.; Chen, C.; Huang, Y. C.; Diao, Z.; Li, S.; Guo, L.; Shen, S. Synergy of Dopants and Defects in Graphitic Carbon Nitride with Exceptionally Modulated Band Structures for Efficient Photocatalytic Oxygen Evolution. Adv. Mater. 2019, 31 (43), 1-10. https://doi.org/10.1002/adma.201903545.

(56) Dong, Y.; Chen, Y.; Jiang, P.; Wang, G.; Wu, X.; Wu, R. A Novel G-C3N4 Based Photocathode for Photoelectrochemical Hydrogen Evolution. RSC Adv. 2016, 6 (9), 74657473. https://doi.org/10.1039/c5ra23265a.

(57) Han, Y. Y.; Lu, X. L.; Tang, S. F.; Yin, X. P.; Wei, Z. W.; Lu, T. B. Metal-Free 2D/2D Heterojunction of Graphitic Carbon Nitride/Graphdiyne for Improving the Hole Mobility 
of Graphitic Carbon Nitride. Adv. Energy Mater. 2018, 8 (16), 1-8.

https://doi.org/10.1002/aenm.201702992.

(58) Hankin, A.; Bedoya-Lora, F. E.; Alexander, J. C.; Regoutz, A.; Kelsall, G. H. Flat Band Potential Determination: Avoiding the Pitfalls. J. Mater. Chem. A 2019, 7 (45), 2616226176. https://doi.org/10.1039/c9ta09569a.

(59) Wang, J.; Zhang, C.; Shen, Y.; Zhou, Z.; Yu, J.; Li, Y.; Wei, W.; Liu, S.; Zhang, Y. Environment-Friendly Preparation of Porous Graphite-Phase Polymeric Carbon Nitride Using Calcium Carbonate as Templates, and Enhanced Photoelectrochemical Activity. J. Mater. Chem. A 2015, 3 (9), 5126-5131. https://doi.org/10.1039/c4ta06778a.

(60) Guo, Y.; Li, J.; Yuan, Y.; Li, L.; Zhang, M.; Zhou, C.; Lin, Z. A Rapid MicrowaveAssisted Thermolysis Route to Highly Crystalline Carbon Nitrides for Efficient Hydrogen Generation. Angew. Chemie - Int. Ed. 2016, 55 (47), 14693-14697. https://doi.org/10.1002/anie.201608453.

(61) Zhang, J.; Zhang, M.; Lin, L.; Wang, X. Sol Processing of Conjugated Carbon Nitride Powders for Thin-Film Fabrication. Angew. Chemie - Int. Ed. 2015, 54 (21), 6297-6301. https://doi.org/10.1002/anie.201501001.

(62) Bellamkonda, S.; Shanmugam, R.; Gangavarapu, R. R. Extending the $\pi$-Electron Conjugation in 2D Planar Graphitic Carbon Nitride: Efficient Charge Separation for Overall Water Splitting. J. Mater. Chem. A 2019, 7 (8), 3757-3771. https://doi.org/10.1039/c8ta10580d.

(63) El-Safty, S. A.; Shenashen, M. A.; Sakai, M.; Elshehy, E.; Halada, K. Detection and Recovery of Palladium, Gold and Cobalt Metals from the Urban Mine Using Novel Sensors/Adsorbents Designated with Nanoscale Wagon- Wheel-Shaped Pores. J. Vis. Exp. 2015, 2015 (106), 53044. https://doi.org/10.3791/53044.

(64) Liu, Y. Is the Free Energy Change of Adsorption Correctly Calculated? J. Chem. Eng. Data 2009, 54 (7), 1981-1985. https://doi.org/10.1021/je800661q.

(65) Achilleos, D. S.; Kasap, H.; Reisner, E. Photocatalytic Hydrogen Generation Coupled to Pollutant Utilisation Using Carbon Dots Produced from Biomass. Green Chem. 2020, 22 (9), 2831-2839. https://doi.org/10.1039/d0gc00318b. 
Table of Contents/Graphical Abstract

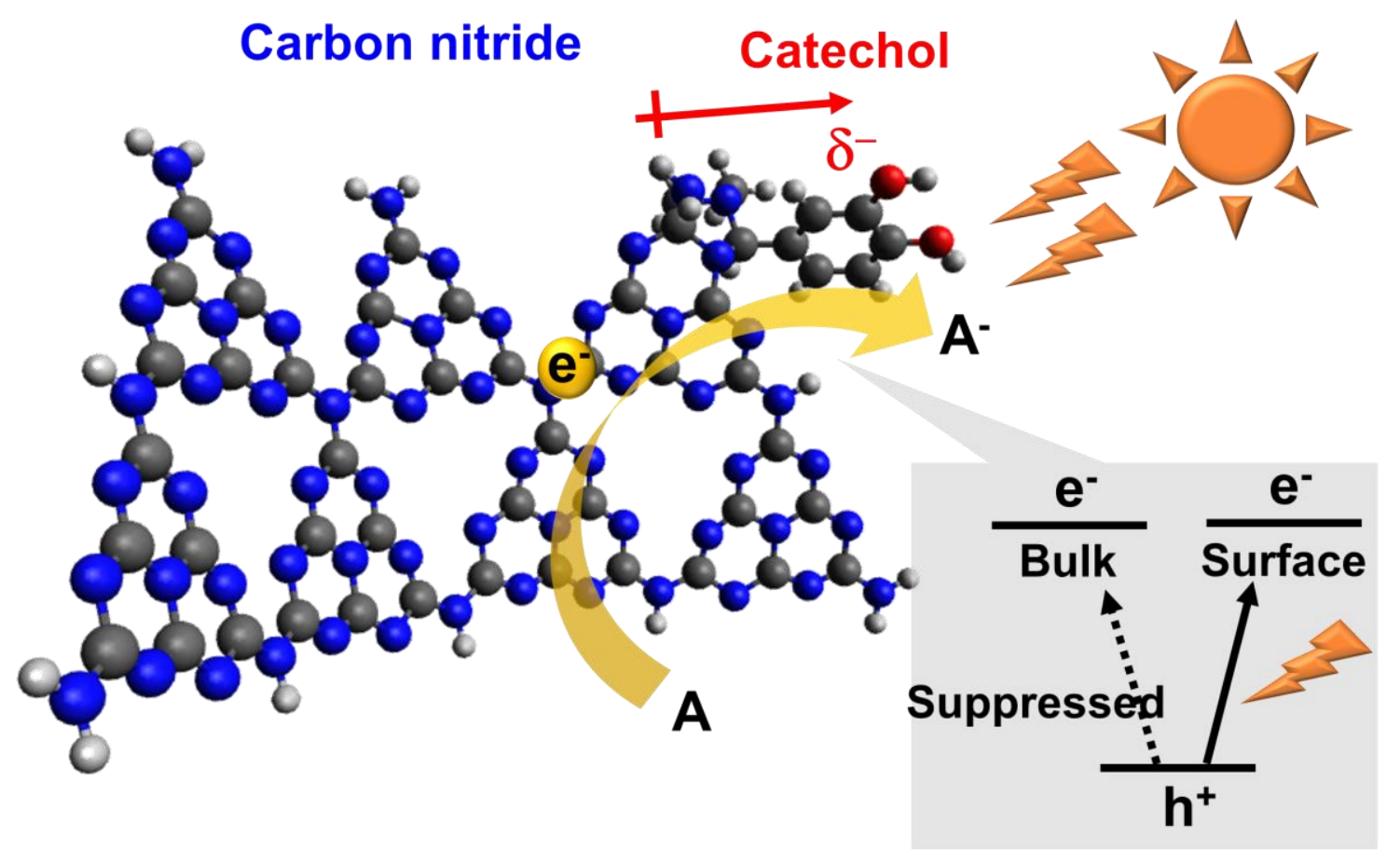

